\title{
Visualizing data visualization: a systematic literature mapping by Brazilian design researchers
}

\author{
Rodrigo Pessoa Medeiros
}

data visualization,

Brazil, design tool, methodology

\begin{abstract}
This paper integrates and expands reflections in the academic Brazilian literature on data visualization from a design perspective between the years 2010 and 2020. It is based on a systematic literature mapping method of 42 articles. The results show the institutions affiliation of researchers and their geographical distribution; the evolution over the years of publications; the methodological approaches and authors cited; the types of studies; and the visualization artifact generated and the design tools employed in this process. Specifically, the results show that The Federal University of Rio de Janeiro (UFRJ) is the institution with the highest concentration of papers and that the southeast region concentrates the most significant number of institutions. The years with the most publications are 2015 and 2019. Although most articles adopt an ad-hoc methodology, two publications use the Ben Fry methodology and its adaptations. Edward Tufte is the most cited author. There is a concentration of authors in the conceptualization of infographics and data visualization. Finally, the creation of artifacts is the most common type of study, Processing is the most popular design tool to create a data visualization, and visualization in Processing is the most frequent result of a study.
\end{abstract}

\section{Introduction}

This paper integrates and expands reflections in the academic Brazilian literature on data visualization. In the last decade, there has been an increase in this theme's interest among designers in Brazil. This is visible in the emergence of numerous initiatives such as academic events (e.g., the Brazilian Congress of Information Design), research groups (e.g., LabVis, Lab DSI, 2ID), community initiatives (e.g., as Dataviz Brasil and dataviz.Rio) and podcasts (e.g., visual+mente). Besides, data visualization has become an important topic in journalism, arts, computing, and statistics. There is also growing interested in Brazilian information technology and design companies in providing data visualization to facilitate complex data analysis.

In light of such emerging debate, there is a particular need for an overview of the field's state. Therefore, this article maps the publications made by Brazilian design researchers on data visualization. The following sections describe the methods and results. 


\section{Methodology}

Systematic literature mapping is an empirical method based on a systematic literature review approach (SLR). Kitchenham \& Charters (2007) confirm the creation of the method in evidence-based software engineering. Through systematic literature mapping, it is possible to identify, interpret, and categorize the studies by research questions or knowledge area (Petersen et al., 2015).

Systematic literature mapping is a formal method characterized by the rigor, systematicity, and reproducibility of science. Therefore, Budgen et al. (2008) recommend systematic literature mapping to identify gaps in current research to suggest areas for further investigation and provide a framework for adequately positioning new research activities. This mapping type is a well-defined methodological process, making the literature results less likely to be skewed. The protocol adopted in this mapping follows the guidelines presented by Kitchenham \& Charters (2007), Petticrew \& Roberts (2008), Budgen et al. (2008), and Petersen et al. (2015) and consists of the following steps:

1. Research questions: Identifying fundamental questions for the review analysis;

2. Selection and exclusion criteria: Defining of explicit inclusion and exclusion criteria for the mapping;

3. Search process: Identifying search protocols (manual or automatic), data sources, and search terms.

4. Selection and Snowballing: Describing all the steps involved in the pre-selection and selection of studies. The snowballing process is also described, which consists of checking the bibliographic references of the articles selected in the search for potential studies to be included in the mapping.

5. Data collection and analysis: Identifying what data was extracted from publications and the form of registration (database, spreadsheet, etc.).

6. Limitations of the protocol: Defining the limitations of the systematic method.

Data visualization is an interdisciplinary area, and it is essential to collaborate with researchers from other areas (e.g., computing, arts, statistics, and geolocation). Therefore, a systematic literature mapping represents an ideal method for revealing research trends about data visualization in design given the heterogeneous nature of the literature.

\section{Systematic literature mapping}

The Systematic Literature Mapping steps are described in this section in the following order: research questions, selection and exclusion 
criteria, search process, selection and snowballing, data collection, and analysis and protocol limitations.

\subsection{Research questions}

This study's central question is "How is data visualization produced from a design perspective in Brazil?". The following specific research questions were defined to answer the central question: RQ1: What are the research affiliation of researchers involved in this area, and how are they distributed geographically?; RQ2: What is the number of studies over the years?; RQ3: What are the methodological approaches and authors cited in the studies?; RQ4: What are the most common types of studies?; RQ5: What is the kind of artifact is the result of a study?; RQ6: What are the most common design tools utilized in the studies?

\subsection{Selection and exclusion criteria}

The review considered articles written in Portuguese or English published in conferences, journals, or book chapters between 2010 and 2020 on data visualization from a design perspective. The articles also needed to contain at least one Brazilian researcher as an author and be relevant to at least three of the research questions outlined above. On the other hand, publications were excluded if they were published only as a summary; address only printed infographics; approach data visualization from the perspective of another related area such as computing, art, etc.; or if a subsequent article derived from the same research already published previously (duplicated studies). In the case of duplicate studies, only the most recent were included.

\subsection{Search process}

For the automatic search process, the search string was assembled from the keywords found in the articles on data visualization in Brazil based on the author's experience as a researcher in the design field. The terms chosen were visualização de dados; visualização; dataviz; data visualization. Therefore, the search string was constructed based on the combination of the terms found, which were concatenated using the Boolean operators "OR" and "AND" and producing the string as the final result: "Visualização" OR "visualização de dados" OR "dataviz" OR "data visualization".

In terms of manual search, the journals "InfoDesign" and "Estudos em Design" were selected since they are the most relevant in the field and therefore central for this review. The manual search process consists of consulting conference proceedings websites and manually accessing all publications, and selecting papers on the basis of their 
titles, abstracts, and keywords. This manual search process included the following conference proceedings: Congresso Internacional de Design da Informação (CIDI), Congresso Nacional de Iniciação Científica em Design da Informação (CONGIC) e Congresso Brasileiro de Pesquisa \& Desenvolvimento em Design (P\&D).

\subsection{Selection and snowballing}

The article selection process involved two stages. In the pre-selection stage, the title, keywords, and abstract of articles were screened according to the inclusion criteria. Then, the full text of the preselected articles was analyzed, and duplicate papers were excluded. The manual search yielded 40 potentially relevant articles, of which 30 were pre-selected. The automatic search provided 20 potentially relevant articles, of which 15 were pre-selected. Together, this yielded a total of 45 pre-selected articles. In the second phase of the selection process, 08 articles from the manual search and 26 articles from the automatic search were selected, totaling 34 relevant articles. Running the snowballing process on these 34 selected articles evidenced 18 other relevant articles, of which 08 were selected. In total, 42 articles were selected for analysis. This process took place between March and September 2020.

\subsection{Data collection and analysis}

The following data were extracted from each study and recorded in the codebook (in spreadsheet format): Search engine (manual, automatic or snowballing) and source (name of the journal, the proceedings of the event or the database, respectively); Title, keywords, authors, type (periodical or proceedings), and year; Methodology; Authors; Design Tool; Type of work (Case study, Theoretical, Artifact creation, Experience report or Experiment) and Result artifact.

A bottom-up inductive approach was adopted to analyze the articles and answer the research question motivating this study. First, the keywords of each article were collected. This revealed different terms used to refer to similar concepts, showing the absence of standard terminology and suggesting that authors freely choose words when discussing a topic. Thus, a list of most common terms consolidating all synonymous keywords. Then related concepts were grouped into categories in each research question. The complete list of selected articles can be viewed at http://bit.ly/3hMOl58. 


\subsection{Protocol limitations}

This study shares the most common shortcomings of the systematic method: limited search coverage and possible biases introduced during study selection, data extraction, and analysis. These limitations were addressed following the general recommendations for systematic mapping - using a combined search between manual and automatic complemented by a snowballing process (Kitchenham \& Charters, 2007; Petticrew \& Roberts, 2008).

\section{Analysis of the results}

This section starts by applying the literature's systematic mapping to analyze literary production regarding data visualization in Brazil and then describes the research questions carried out among the 42 selected articles.

\subsection{Research institutions involved with data visualization (RQ1)}

During the period under review, 16 Brazilian research institutions published articles on data visualization. Some of these publications were made in partnership with 6 international research institutions. Among the articles analyzed, some were written based on partnerships between authors from different Brazilian and international institutions, such as Harvard University and University of the Arts London.

Of the 42 articles analyzed, 30 were published by authors from a single institution, 7 had authors from two different institutions, and 3 had authors from 3 different institutions. Table 1 presents the articles analyzed according to their author's affiliation. It is clear that some institutions have a greater concentration of published works, such as the Federal University of Rio de Janeiro, which features 11 publications, many with Professor Doris Kosminsky participation.

Another example is the 5 publications from the State University of Rio de Janeiro made at the Superior School of Industrial Design (ESDI). As well as those at the Federal University of Espírito Santo, which had a significant impact due to Professor Dr. Mauro Pinheiro's work at both institutions.

The three publications at Northeastern University are also noteworthy. They refer to the work of Professor Isabel Meirelles. The latter contributed to the internationalization process of data visualization research by cooperating with other institutions, such as Harvard University and Massachusetts Institute of Technology, in the United States, and with Tongji University in China. 
Table 1 Publication of articles by the institution.

\begin{tabular}{|c|c|}
\hline Institution & Number of articles published \\
\hline Universidade Federal do Rio de Janeiro (UFRJ) & 11 \\
\hline Universidade Estadual do Rio de Janeiro (UERJ) & 05 \\
\hline Universidade Federal do Espírito Santo (UFES) & 05 \\
\hline Universidade de Brasília (UnB) & 05 \\
\hline Universidade de São Paulo (USP) & 05 \\
\hline Northeastern University & 03 \\
\hline Universidade Anhembi Morumbi & 03 \\
\hline Instituto Federal da Paraíba (IFPB) & 02 \\
\hline Pontifícia Universidade Católica do Rio de Janeiro (PUC-Rio) & 02 \\
\hline Universidade Federal de Santa Catarina (UFSC) & 02 \\
\hline Bauhaus-Universität Weimar & 01 \\
\hline Fundação Getulio Vargas (FGV-Rio) & 01 \\
\hline Harvard University & 01 \\
\hline Instituto Brasileiro de Informação em Ciência e Tecnologia & 01 \\
\hline Massachusetts Institute of Technology (MIT) & 01 \\
\hline Tongji University & 01 \\
\hline Universidade Federal de Pernambuco (UFPE) & 01 \\
\hline Universidade Federal de Pelotas (UFPel) & 01 \\
\hline UniBrasil & 01 \\
\hline Universidade Positivo & 01 \\
\hline University of the Arts London & 01 \\
\hline
\end{tabular}

\subsubsection{Distribution of publications by region of Brazil (RQ1)}

Analyzing the distribution of articles published by region of the country reveals some geographic differences (see Table 2). Specifically, analyzing articles by region, it is noteworthy that, in the last decade, there were no publications in the northern region of Brazil.

Table 2 Articles published by region of Brazil.

\begin{tabular}{lc}
\hline Region of Brazil & Number of articles published \\
\hline Southeast & 31 \\
Midwest & 06 \\
South & 06 \\
Northeast & 03 \\
North & 00 \\
\hline
\end{tabular}


On the other hand, there is a concentration of articles in the southeastern region of Brazil. This region alone is responsible for more than $67 \%$ of the production of data visualization in the country. Further analyzing the articles published in the southeastern region in more detail reveals another discrepancy in the intellectual production on this theme, as shown in Table 3. Namely, that the state of Rio de Janeiro is responsible for more than $58 \%$ of publications in this region.

Table 3 Articles published in the Southeast region of Brazil.

\begin{tabular}{lc}
\hline States of Southeast region of Brazil & Number of articles published \\
\hline Rio de Janeiro & 18 \\
São Paulo & 08 \\
Espírito Santo & 05 \\
Minas Gerais & 00 \\
\hline
\end{tabular}

\subsection{Evolution of the number of articles over the years (RQ2)}

The chronology of the articles is shown in Table 4. During this period, there were 30 publications in conference proceedings and 12 in journals. Specifically, the number of publications was higher in 2011, 2015, 2017, and 2019-the years when the Congresso Internacional de Design da Informação (CIDI) took place.

Table 4 Chronology of publications.

\begin{tabular}{lc}
\hline Years & Number of articles published \\
\hline 2010 & 02 \\
2011 & 06 \\
2012 & 03 \\
2013 & 01 \\
2014 & 03 \\
2015 & 09 \\
2016 & 01 \\
2017 & 05 \\
2018 & 03 \\
2019 & 09 \\
\hline
\end{tabular}

\subsection{Methodologies, authors, and topics used in the studies (RQ3)}

In this subsection, the results from the review were organized by methodological approach, authors cited, and the topics most covered. 


\subsubsection{Methodologies used in the studies (RQ3)}

The methods used by the analyzed articles is shown in Table 5. Most articles do not use any particular method for data collection, data analysis, and the creation of data visualizations. While most articles do not reference whether the data visualization process was based on any established methodological approach, two publications describe using the method developed by Ben Fry (2007). This method has the following steps: extract the data; analyze them; filter them according to design goals; mine them; represent them in some visual way; refine presentation; and, finally, add elements to facilitate interaction with this data.

Two articles also describe using the user-centered design philosophy (Rogers, Sharp, \& Preece, 2008), even though this method is primarily used in user experience design (UX Design) to create digital artifacts such as websites and applications. Another method with primary use in UX Design mentioned in an article is the double diamond, developed by the British Design Council (Design Council, 2015). A physical data visualization publication mentioned the constructive visualization method proposed by Huron et al. (2014).

Other relevant methods in the area of infographics appear in a handful of articles. Specifically, idX - Information Design by Core Competences (2007), the method $\langle 6\rangle\langle 6\rangle$ by Roam (2013), and materials from Cairo (2011) and Sless (2004). Also mentioned are Steele \& Iliinsky (2010) process for data visualization and the Ricardo Cunha Lima (2009) method on the use of graphic elements applied to journalistic infographics.

Table 5 Methodologies used in studies.

\begin{tabular}{lcl}
\hline Methodologies & Number of articles & Primary use \\
\hline Ad-hoc model & 09 & Data visualization \\
User centered design & 02 & UX Design \\
Ben Fry's method (2007) and adaptations & 02 & Data visualization \\
Methodology adapted from Steele and lliinsky (2010) & 01 & Data visualization \\
Methodology adapted from Cairo (2011) & 01 & Infographics \\
Roam's method $<6><6>$ (2013) & 01 & Infographics \\
Methodology adapted from Sless (2004) & 01 & Infographics \\
Core Competences idx methodology (2007) & 01 & Infographics \\
Use of the Ricardo Cunha Lima method (2009) & 01 & Infographics \\
Constructive visualization methodology by Huron et al. (2014) & 01 & Physical data visualization \\
Double Diamond Methodology & 01 & UX Design \\
\hline
\end{tabular}




\subsubsection{Authors cited in the studies (RQ3)}

The authors most cited in the publications are shown in Table 6. This study choice was to consider only authors who had three or more citations. The three most-cited authors were Edward Tufte, Alberto Cairo, and Lev Manovich. Four Brazilian authors are among the most cited. Namely, Isabel Meirelles, Mauro Pinheiro, Priscila Farias, and Ricardo Cunha Lima. The first one has six citations, the most citations among Brazilian authors. Among the most cited authors, there are renowned researchers from the design as well as related areas such as computing and communication. This confirms that the topics covered in the articles reviewed are considerably interdisciplinary.

Table 6 Authors most cited in studies.

\begin{tabular}{lc}
\hline Authors & Number of citations \\
\hline Edward Tufte & 16 \\
Alberto Cairo & 13 \\
Lev Manovich & 13 \\
Stuard Card & 10 \\
Jacques Bertin & 08 \\
Michael Twyman & 06 \\
Yuri Engelhardt & 06 \\
Robert Horn & 06 \\
Isabel Meirelles & 06 \\
Richard Wurman & 06 \\
Colin Ware & 05 \\
William Cleveland & 04 \\
Robert Spence & 04 \\
Steven Few & 04 \\
Jorge Frascara & 04 \\
Nathan Yau & 04 \\
Jeanne Preece & 04 \\
Edward Segel & 04 \\
Donald Norman & 03 \\
Mauro Pinheiro & 03 \\
Priscila Farias & 03 \\
Ricardo Cunha Lima & \\
Mark Weiser & 03 \\
\hline & \\
& 04 \\
\hline
\end{tabular}

\subsubsection{Topics covered in the studies (RQ3)}

The main areas addressed in the analyzed articles were categorized and are represented in Figure 1. The topic with most references is the conceptualization of infographics and data visualization with a 
1 The complete references of the authors cited in Figure 1 are available at https://bit.ly/3259zEf total of thirteen appearances. Graphic language, cognition, and visual metaphors appear with seven references; and the environmental information system with six references. Data journalism, and graphic representations have four references. Then how to do data visualization and narratives (storytelling) have four references. Visualization of physical data and visual perception have three each. The topics of data visualization related to the history of design and information anxiety had two references. Finally, the topics democratize information, visual literacy, visualization of synesthetic data, content analysis, identification of absences, and UX Design had only one reference.

Figure 1 Topics and their references. ${ }^{1}$

\begin{tabular}{|c|c|}
\hline Topics & References \\
\hline $\begin{array}{l}\text { Conceptualization infographics and } \\
\text { data visualization }\end{array}$ & $\begin{array}{l}\text { Tufte (1983); Card, Mackinlay, \& Sheneiderman (1999); Shedroff (2000); } \\
\text { Engelhardt (2002); Kosara (2007); Few (2009); Cairo (2008; 2011); Bertin (2010); } \\
\text { Manovich (2010); Frascara (2011); Meirelles (2011); Ciuccarelli (2012). }\end{array}$ \\
\hline Graphic Language & Twyman (1979); Engelhardt (2002); Lima (2009; 2015); Meirelles (2013). \\
\hline Cognition and visual metaphors & $\begin{array}{l}\text { Lakoff (1987); Norman (1993); Gershon (1992); Card et al (1999); Ware (2004); } \\
\text { Meirelles (2007); Lima (2018). }\end{array}$ \\
\hline Ambient information systems & $\begin{array}{l}\text { Pinheiro (2010 and 2011); Weiser \& Brown (1997); Weiser, Brown, \& Gold (1999); } \\
\text { Hazlewood, Coyle, and Consolvo (2007); Mankoff \& Dey (2003). }\end{array}$ \\
\hline Data journalism & $\begin{array}{l}\text { Peltzer (1991); Cairo (2008); Miranda \& Spinillo (2012); Miranda (2013); } \\
\text { Giannella (2014). }\end{array}$ \\
\hline Graphical representations & $\begin{array}{l}\text { Horn (1998); Bertin (2010); Lankow, Ritchie, \& Crooks (2012); Miranda, Spinillo \& } \\
\text { Fontoura (2012); Andrade (2014). }\end{array}$ \\
\hline How to do data visualization & Spence (2007); Fry (2008); Steele and Illinsky (2010); Yau (2013). \\
\hline Storytelling & Segel and Heer (2010); Munzer (2014); Knaflic (2015); Tufte (2016). \\
\hline $\begin{array}{l}\text { The physicality of Information } \\
\text { Visualization }\end{array}$ & Lau \& Vande Moere (2007); Vande Moere (2008); Jansen, Dragicevic, \& Fekete (2013). \\
\hline Visual perception & Healy et al. (1996); Lima (2005); Healy (2017). \\
\hline Data visualization and design history & Farias (2019); Farias, Hanns, \& Dixon (2016). \\
\hline Information anxiety & Wurman (1991); Richtel (2010). \\
\hline Democratization of data visualization & Viegas et al. (2007). \\
\hline Visual literacy & Horn (1998). \\
\hline Visualization of synesthetic data & Cox (2014). \\
\hline Content analysis & Krippendorff (2004). \\
\hline Absence identification & Floridi (2011). \\
\hline UX Design & Preece, Rogers, \& Sharp (2008). \\
\hline
\end{tabular}

\subsection{Types of studies (RQ4)}

Regarding the types of studies, Table 7 shows the differences among publications. Specifically, twenty-one articles focus on the creation of artifacts, eleven focus on data studies, six are theoretical, three are 
experience reports (in general teaching), and one has an experimental approach. The first category stands out because it represents a promising number of works developed in that period.

Table 7 Type of studies.

\begin{tabular}{lc}
\hline Type of studies & Number of articles \\
\hline Artifact creation & 21 \\
Case study & 11 \\
Theoretical & 06 \\
Experience report & 03 \\
Experiment & 01 \\
\hline
\end{tabular}

\subsection{Types of artifact used as a result (RQ5)}

The artifacts presented as a result of the studies are shown in Table 8. This includes a column with the type of study from Table 7. Among the articles which focus on artifact creation, visualization of data in Processing is the most common and appears in five publications. Four articles featured functional prototypes. Static maps or cartograms, interactive PDF, physical visualization, and websites appear in two articles. Finally, the following artifacts were mentioned in only one article: robot on Twitter, animated videos, graphs on Gephi, visualizations on Tableau, and visualization in javascript in the browser. A created artifact was added as Interactive PDF and Maps or static cartogram.

In the articles that carried out a case study, five used data visualization four used infographics, and two included ambient information systems. An article also created a comparative table on the use of time in visual representations. Among experience reports articles, two feature curators by the lectures for the event, one shows the creation of a methodology for making infographics. Finally, a theoretical article featured a list of the types of visual representations.

\subsection{Tools used in studies (RQ6)}

The design tools for data visualization are shown in Table 9. The most used design tool was Processing, and it was mentioned in five articles. Processing is a tool for creative programming and is widely used in the creation of data visualization. Adobe Indesign and ManyEyes were tools cited in two studies. Adobe Indesign was used to create interactive PDFs. ManyEyes (Viegas et al., 2007) was a platform created, currently deactivated, by IBM in the team led by Brazilian Fernanda Viegas and Martin Wattenberg. Other tools cited by at least 
Table 8 Type of artifact used as a result in studies.

\begin{tabular}{lcl}
\hline An artifact used as a result & Number of articles & Type of study \\
\hline Data visualization in Processing & 05 & Artifact creation \\
Functional prototypes & 04 & Artifact creation \\
Static maps or cartograms & 02 & Artifact creation \\
Interactive PDF & 02 & Artifact creation \\
Physical visualization & 02 & Artifact creation \\
Websites & 02 & Artifact creation \\
Twitter robots & 01 & Artifact creation \\
Animated videos & 01 & Artifact creation \\
Graphs in Gephi & 01 & Artifact creation \\
Data visualization in Tableau & 01 & Artifact creation \\
Data visualization in javascript & 01 & Artifact creation \\
Data visualization case study & 05 & Case study \\
Infographics case study & 04 & Case study \\
Ambient information systems case study & 02 & Case study \\
A comparative chart on the use of time & 01 & Case study \\
in visual representation & & \\
Curator of lectures for event & 02 & Experience report \\
Creation of the methodology for & & Experience report \\
creating infographics & & \\
List with the types of visual representations: & & Theoretical \\
maps, statistical graphs, network diagrams, \\
statistical maps, network maps, statistical \\
network diagram, statistical network maps
\end{tabular}

one article, with different focuses, include: creating the mind map (freemind, ideographer, iMap for inventor, InfoRapid, Thortspace), connection for physical artifacts (Arduino), prototyping for digital interfaces (Figma), georeferenced maps (GIS), graph (Gephi), creative programming (Javascript), content analysis (MAXQDA), tabulated data (CSV, Google Spreadsheets, Microsoft Excel), layer overlay (Adobe Photoshop), vectorization (Adobe Illustrator), image preview in large size (Closr), data collection (Python), database (PostgreSQL), word cloud (visuWords and Wordle.com), timeline (xTimeLine.com) and data visualization (RAW Graphs and Tableau).

\section{Conclusion}

A systematic mapping of the literature was carried out in 42 articles to improve the understanding of data visualization from a design perspective in Brazil. This article aims to both stimulate this debate by mapping the topics covered by these publications and thus outline relevant questions for future research in the area. 
Table 9 Tools mentioned in the studies.

\begin{tabular}{|c|c|c|}
\hline Tools & Number of articles & Goal \\
\hline Processing & 05 & Creative programming \\
\hline Adobe Indesign & 02 & Interactive PDF \\
\hline Manyeyes & 02 & Data visualization \\
\hline Arduino & 01 & Connection for physical artifacts \\
\hline Adobe Illustrator & 01 & Vector drawing \\
\hline Adobe Photoshop & 01 & Photomontage \\
\hline Closr & 01 & View large images \\
\hline CVS & 01 & Tabulated data \\
\hline Figma & 01 & Prototype for digital interfaces \\
\hline Freemind & 01 & Mindmap \\
\hline Geographic Information System (GIS) & 01 & Georeferenced maps \\
\hline Gephi & 01 & Graph visualization \\
\hline Google Sheets & 01 & Tabulated data \\
\hline Ideographer & 01 & Mindmap \\
\hline iMap for inventor & 01 & Mindmap \\
\hline InfoRapid & 01 & Mindmap \\
\hline Javascript & 01 & Creative programming \\
\hline MAXQDA & 01 & Content analysis \\
\hline Microsoft Excel & 01 & Tabulated data \\
\hline Python & 01 & Data collect \\
\hline PostgreSQL & 01 & Database \\
\hline RAW Graphs & 01 & Data visualization \\
\hline Tableau & 01 & Data visualization \\
\hline Thortspace & 01 & Mindmap \\
\hline VisuWords & 01 & Word cloud \\
\hline Wordle.com & 01 & Word cloud \\
\hline XTimeLine.com & 01 & Timeline \\
\hline
\end{tabular}

Regarding RQ1, the Federal University of Rio de Janeiro (UFRJ) was the institution with the highest concentration of articles reflecting the research concentration in the southeast region of Brazil. There is no article from institutions in the country's northern region from 2010 to 2020. Moreover, the international collaboration of researchers stands out and involves eight institutions abroad. In this context, Professor Isabel Meirelles seems to have a particular role in these international partnerships. As a result of RQ2, the years 2015 and 2019 had the highest number of publications, nine in each. In this regard, it is evident that the years with the greatest concentration of works coincide with the realization of CIDI in the country.

Regarding RQ3, although nine articles seem to use an ad hoc methodology, without providing any detailed description, two 
publications used the Ben Fry methodology (2008) and its adaptations. There are also citations to the methods of Cairo (2011) and Steele and Illinsky (2010). Edward Tufte $(1983,2016)$ was the most cited author with sixteen appearances, accompanied by Alberto Cairo $(2008,2011)$ and Lev Manovich (2010), which appear in thirteen articles. There is also a clear concentration of authors in the conceptualization of infographics and data visualization and the topics of graphic language, cognition, and visual metaphors.

About RQ4, the creation of artifacts is the type of study most common with twenty-one publications, followed by a case study carried out in eleven studies. Regarding RQ5, visualization in Processing is the most common artifact produced in studies, appearing in five publications (four publications of which made functional prototypes). Finally, in RQ6, Processing also appeared as the most used design tool.

Some considerations need to be made when analyzing the results. The number of Brazilian authors on data visualization is still tiny, and the production in Portuguese is similarly limited. Greater incentive is needed for institutions and research groups to stimulate the theme among design researchers. This is relevant for the South, Midwest, North, and Northeast regions and particularly important for the North region. It also seems that the design tools for creating data visualization are constantly changing. Some of those cited in the articles are not even common today. Although the type of work most common in the articles analyzed is the creation of data visualization, the Brazilian national production on this theme is still little known worldwide. This highlights the need for further diffusing at an international scale the studies and projects carried out in the country.

\section{Acknowledgment}

The author would like to thank Dr. Pedro Monteiro for reviewing and carefully reading the manuscript.

\section{References}

Budgen, D., Turner, M., Brereton, P., \& Kitchenham, B. (2008). Using Mapping Studies in Software Engineering. In Buckley, J., Rooksby, J., \& Bednarik, R. Proceedings of the 2oth Annual Meeting of the Psychology of Programming Interest Group. Lancaster: Lancaster University.

Cairo, A. (2008). Infografia 2.o: visualización interactiva de información en prensa. Madrid: Alamut.

Cairo, A. (2011). El Arte Funcional. Infografia y visualización de información. Madrid: Alamut. 
Design Council. (2021, Jan 12). The Design Process: What is the Double Diamond? Retrieved from https://www.designcouncil.org.uk/news-opinion/ design-process-what-double-diamond.

Fry, Ben. (2008). Visualizing Data. Sebastopol, CA: O’Reilly Media.

Huron, S., Carpendale, S., Thudt, A., Tang, A., \& Maurer, M. (2014). Constructive visualization. In Proceedings of the 2014 Conference on Designing Interactive Systems - DIS 14 (pp. 433-442), doi:10.1145/2598510.2598566.

idX - Information Design - Core Competences. (2007, Aug 31). What information designers know and can do. Retrieved from http://www.iiid.eu/ idx-information-design-core-competencies.

Kitchenham, B., Charters, S. (2017). Guidelines for performing systematic literature reviews in software engineering. Keele, United Kingdom.

Manovich, Lev. (2010, Oct). What is visualization? Retrieved from http:// manovich.net/index.php/projects/what-is-visualization

Petersen, K., Vakkalanka, S., \& Kuzniarz, L.. (2015). Guidelines for conducting systematic mapping studies in software engineering: An update. In Information and Software Technology, (pp. 1-18), 64.

Petticrew, M., Roberts, H. (2008). Systematic Reviews in the Social Sciences: a practical guide. Hoboken, NJ, USA: Blackwell Publishing.

Preece, J., Rogers, Y., \& Sharp, H. (2008). Design de interação: além da interação homem-computador. Porto Alegre, Brazil: Bookman.

Roam, D. (2013). The Back of the Napkin: Solving Problems and Selling Ideas with pictures. Portfolio Trade.

Sless, D. (2004, Dec). Labelling code of practice: designing usable non-prescription medicine labels for consumers. Retrieved from: www.communication.org.au.

Steele, J., Ilinsky, N. (2010). Beautiful Visualization. California: O’Reilly Media.

Tufte, E. R. (1983). The Visual Display of Quantitative Information. Cheshire: Graphics Press.

Tufte, E. R. (2016). Beautiful Evidence. Cheshire, CN: Graphics Press.

Viegas F. B., Wattenberg, M., Ham, F. V., Kriss, J., \& Mckeon, M. (2007).

ManyEyes: A Site for Visualization at Internet Scale. IEEE Transactions on Visualization and Computer Graphics, 13(6), (pp. 1121-1128).

\section{About the author}

Rodrigo Pessoa Medeiros

rodrigo.medeiros@ifpb.edu.br

Instituto Federal da Paraíba, campus Cabedelo, Brasil

Submission date: $11 / 4 / 2021$

Approvement date: 28/8/2021 